\title{
HAT-P-12b: A LOW-DENSITY SUB-SATURN MASS PLANET TRANSITING A METAL-POOR K DWARF*
}

\author{
J. D. Hartman ${ }^{1}$, G. Á. Bakos ${ }^{1,10}$, G. Torres ${ }^{1}$, Géza Kovács ${ }^{2}$, R. W. Noyes ${ }^{1}$, A. Pál ${ }^{1,2,3}$, D. W. LAtham ${ }^{1}$, B. Sipő́cz ${ }^{1,3}$, \\ D. A. Fischer ${ }^{4}$, J. A. Johnson ${ }^{5}, 11$, G. W. Marcy ${ }^{6}$, R. P. Butler ${ }^{7}$, A. W. Howard ${ }^{6}$, G. A. Esquerdo ${ }^{1}$, D. D. SAsSelov ${ }^{1}$, \\ GÁbor Kovács ${ }^{1}$, R. P. StefaniK ${ }^{1}$, J. M. Fernandez ${ }^{1,8}$, J. LÁzÁR ${ }^{9}$, I. PAPP ${ }^{9}$, AND P. SÁri ${ }^{9}$ \\ ${ }^{1}$ Harvard-Smithsonian Center for Astrophysics, Cambridge, MA, USA; gbakos@cfa.harvard.edu \\ ${ }^{2}$ Konkoly Observatory, Budapest, Hungary \\ ${ }^{3}$ Department of Astronomy, Eötvös Loránd University, Budapest, Hungary \\ ${ }^{4}$ Department of Physics and Astronomy, San Francisco State University, San Francisco, CA, USA \\ ${ }^{5}$ Institute for Astronomy, University of Hawaii, Honolulu, HI 96822, USA \\ ${ }^{6}$ Department of Astronomy, University of California, Berkeley, CA, USA \\ ${ }^{7}$ Department of Terrestrial Magnetism, Carnegie Institute of Washington, DC, USA \\ ${ }^{8}$ Department of Astronomy, Pontificia Universidad Católica, Santiago, Chile \\ ${ }^{9}$ Hungarian Astronomical Association, Budapest, Hungary \\ Received 2009 April 29; accepted 2009 October 13; published 2009 November 5
}

\begin{abstract}
We report on the discovery of HAT-P-12b, a transiting extrasolar planet orbiting the moderately bright $V \approx 12.8 \mathrm{~K} 4$ dwarf GSC 03033-00706, with a period $P=3.2130598 \pm 0.0000021 \mathrm{~d}$, transit epoch $T_{c}=2454419.19556 \pm 0.00020$ (BJD), and transit duration $0.0974 \pm 0.0006 \mathrm{~d}$. The host star has a mass of $0.73 \pm 0.02 M_{\odot}$, radius of $0.70_{-0.01}^{+0.02} R_{\odot}$, effective temperature $4650 \pm 60 \mathrm{~K}$, and metallicity $[\mathrm{Fe} / \mathrm{H}]=-0.29 \pm$ 0.05 . We find a slight correlation between the observed spectral line bisector spans and the radial velocity, so we consider, and rule out, various blend configurations including a blend with a background eclipsing binary, and hierarchical triple systems where the eclipsing body is a star or a planet. We conclude that a model consisting of a single star with a transiting planet best fits the observations, and show that a likely explanation for the apparent correlation is contamination from scattered moonlight. Based on this model, the planetary companion has a mass of $0.211 \pm 0.012 M_{\mathrm{J}}$ and radius of $0.959_{-0.021}^{+0.029} R_{\mathrm{J}}$ yielding a mean density of $0.295 \pm 0.025 \mathrm{~g} \mathrm{~cm}^{-3}$. Comparing these observations with recent theoretical models, we find that HAT-P-12b is consistent with a $\sim 1-4.5 \mathrm{Gyr}$, mildly irradiated, $\mathrm{H} / \mathrm{He}$-dominated planet with a core mass $M_{C} \lesssim 10 M_{\oplus}$. HAT-P-12b is thus the least massive $\mathrm{H} / \mathrm{He}$-dominated gas giant planet found to date. This record was previously held by Saturn.
\end{abstract}

Key words: planetary systems - stars: individual (HAT-P-12, GSC 03033-00706) - techniques: photometric techniques: spectroscopic

Online-only material: color figures, machine-readable table

\section{INTRODUCTION}

Transiting extrasolar planets (TEPs) provide unique opportunities to study the physical properties of planetary mass objects outside the solar system. By combining time-series photometric observations taken during transit with radial velocity (RV) measurements of the star, it is possible to precisely measure the mass and radius of the planet, if the stellar mass and radius can be determined by other means. The bulk density of the planet may then be compared with the predictions of theoretical planetary structure models (e.g., Baraffe et al. 2008; Fortney et al. 2007; Burrows et al. 2007; Seager et al. 2007) to infer the structure of the planet. Discoveries of planets that fall outside the predicted mass-radius range (e.g., inflated hot Jupiters such as TrES-4b; Mandushev et al. 2007) lead in turn to refinements of these models. TEPs also provide unique opportunities to study planetary atmospheres, including their composition (e.g., Charbonneau et al. 2002) and their thermal profiles (e.g., Knutson et al. 2008). It is also possible to measure the projection of the angle between the orbital axis and the stellar spin

\footnotetext{
* Based in part on observations obtained at the W. M. Keck Observatory, which is operated by the University of California and the California Institute of Technology. Keck time has been granted by NOAO (A264Hr, A146Hr) and NASA (N162Hr, N128Hr).

${ }^{10}$ NSF Fellow.

${ }^{11}$ NSF Postdoctoral Fellow.
}

axis for these planets (e.g., Winn et al. 2005), which may be used to test theories of planetary migration (Fabrycky \& Winn 2009).

To date more than 40 TEP discoveries have been published, with the majority coming from dedicated photometric surveys. ${ }^{12}$ These planets span a range covering more than 2 orders of magnitude in mass from a Super-Earth TEP (Corot-7b; Léger et al. 2009) and Super-Neptunes (GJ 436b and HAT-P-11b; Gillon et al. 2007; Bakos et al. 2009b) to brown dwarf size objects (Corot-3b, XO-3b; Deleuil et al. 2008; Johns-Krull et al. 2008). Focusing on the low-mass end, we note that the three least massive TEPs with well determined masses (GJ 436b, HAT-P11b, and HD 149026b; Sato et al. 2005), are also the three TEPs with the smallest radii (excluding Corot- $3 b$ ) and highest inferred core mass fractions. Above this, we begin to see planets with a wide range of radii. The planets WASP-11/HAT-P-10b (Bakos et al. 2009a; West et al. 2009a), WASP-6b (Gillon et al. 2009), HAT-P-1b (Bakos et al. 2007b), OGLE-TR-111b (Pont et al. 2004), WASP-15b (West et al. 2009b), and XO-2b (Burke et al. 2007) all have radii larger than or comparable to that of Jupiter, whereas HAT-P-3b (Torres et al. 2007, 2008) has a radius that is only slightly larger than that of Saturn. Given the small number of TEPs known with $M \lesssim 0.5 M_{\mathrm{J}}$, we cannot yet say what is the empirical minimum mass of coreless, or

\footnotetext{
12 http://www.exoplanet.edu/catalog-transit.php
} 
envelope-dominated, gas giant planets. To explore the possible transition from envelope-dominated to core-dominated planets, it is necessary to find more low-mass TEPs.

The Hungarian-made Automated Telescope Network (HATNet; Bakos et al. 2004) survey has been a major contributor to the discovery of TEPs. Operational since 2003, it has covered approximately $10 \%$ of the Northern sky, searching for TEPs around bright stars $(8 \lesssim I \lesssim 12.5 \mathrm{mag})$. HATNet operates six wide field instruments: four at the Fred Lawrence Whipple Observatory (FLWO) in Arizona, and two on the roof of the Submillimeter Array hangar (SMA) of SAO in Hawaii. Since 2006, HATNet has announced and published 11 TEPs. In this work, we report on the 12th such discovery. This planet is only the fourth sub-Saturn mass TEP announced to date, but unlike the other planets, it is of low density, and appears to be $\mathrm{H} / \mathrm{He}$ dominated.

The structure of the paper is as follows. In Section 2 we summarize the observations, including the photometric detection, and follow-up observations. In Section 3 we describe the analysis of the data, such as the stellar parameter determination (Section 3.1), blend modeling (Section 3.2), and global modeling of the data (Section 3.3). We discuss our findings in Section 4.

\section{OBSERVATIONS}

\subsection{Photometric Detection}

The transits of HAT-P-12b were detected with the HAT-5 telescope in Arizona. The region around GSC 03033-00706, a field internally labeled as 145 , was observed on a nightly basis between 2006 January and July, whenever weather conditions permitted. We gathered 4205 exposures of 5 minutes at a 5.5 minute cadence, of which 2927 images were used in the final light curve of HAT-P-12. Each image contained approximately 10,000 stars down to $I \sim 14.0$. For the brightest stars in the field, we achieved a per-image photometric precision of $3 \mathrm{mmag}$.

The calibration of the HATNet frames was done utilizing standard procedures. The calibrated frames were then subjected to star detection and astrometry, as described in Pál \& Bakos (2006). Aperture photometry was performed on each image at the stellar centroids derived from the Two Micron All Sky Survey (2MASS) catalog (Skrutskie et al. 2006) and the individual astrometrical solutions. The resulting light curves were decorrelated against trends using the External Parameter Decorrelation technique in "constant" mode (EPD; see Bakos et al. 2009b) and the Trend Filtering Algorithm (TFA; see Kovács et al. 2005). The light curves were searched for periodic box-like signals using the Box Least-Squares method (BLS; see Kovács et al. 2002). We detected a significant signal in the light curve of GSC 03033-00706 (also known as 2MASS $13573347+4329367 ; \alpha=13^{\mathrm{h}} 57^{\mathrm{m}} 33^{\mathrm{s}} .48, \delta=+43^{\mathrm{d}} 29^{\mathrm{m}} 36^{\mathrm{s}} .7$; J2000; $V=12.84 \pm 0.09$; Droege et al. 2006), with a depth of $\sim 20 \mathrm{mmag}$, and a period of $P=3.2131$ days. The dip had a relative duration (first to last contact) of $q \approx 0.0303 \pm 0.0002$, corresponding to a total duration of $P q \approx 2.337 \pm 0.015 \mathrm{hr}$ (see Figure 1).

\subsection{Reconnaissance Spectroscopy}

All HATNet candidates are subjected to thorough investigation before using more precious time on large telescopes. One of the important tools for establishing whether the transit-like feature in the light curve of a candidate is due to astrophysical

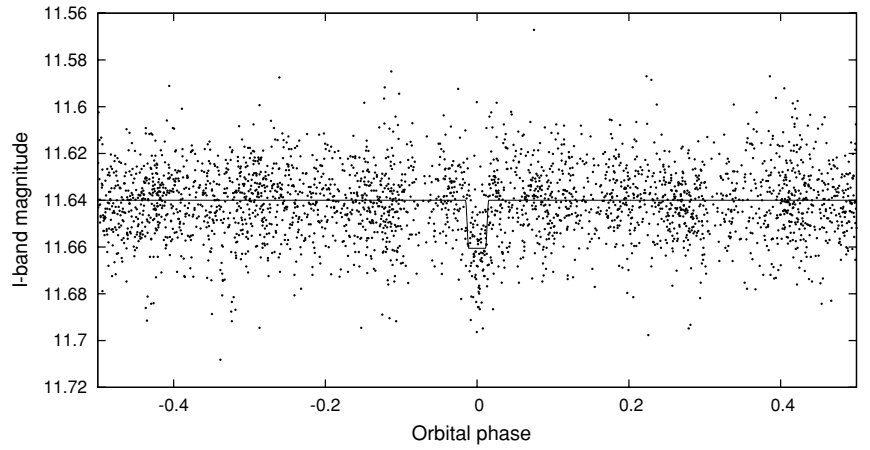

Figure 1. Unbinned light curve of HAT-P-12 including all 2927 instrumental I-band 5.5 minute cadence measurements obtained with the HAT-5 (Arizona) telescope of HATNet (see the text for details), and folded with the period of $P=3.2130598$ days (which is the result of the fit described in Section 3). The solid line shows a boxcar transit model fit to the light curve.

phenomena other than a planet transiting a star is the CfA Digital Speedometer (DS; Latham 1992), mounted on the FLWO $1.5 \mathrm{~m}$ telescope. This yields high-resolution $(R=35,000) \mathrm{spec}-$ tra with low signal-to-noise ratio sufficient to derive RVs with moderate precision (roughly $0.5-1 \mathrm{~km} \mathrm{~s}^{-1}$ ), and to determine the effective temperature and surface gravity of the host star. With this facility we are able to reject many types of false positives, such as F dwarfs orbited by M dwarfs, grazing eclipsing binaries, triple and quadruple star systems, or giant stars where the transit signal cannot be due to a planet.

We obtained eight observations of HAT-P-12 with the DS. The RV measurements of HAT-P-12 showed an rms residual of $0.43 \mathrm{~km} \mathrm{~s}^{-1}$, consistent with no detectable RV variation. The spectra were single-lined, showing no detectable evidence for more than one star in the system. Atmospheric parameters for the star, including the effective temperature $T_{\text {eff } \star}=4500 \pm$ $250 \mathrm{~K}$, surface gravity $\log g_{\star}=4.0 \pm 0.2$ (log cgs), and projected rotational velocity $v \sin i$ consistent with zero with an asymmetric error of about $1 \mathrm{~km} \mathrm{~s}^{-1}$, were derived as described by Torres et al. (2002). The effective temperature and surface gravity correspond to a mid-K dwarf. The mean heliocentric RV of HAT-P-12 is $-40.51 \pm 0.21 \mathrm{~km} \mathrm{~s}^{-1}$.

\subsection{High-Resolution, High-S/N Spectroscopy}

Given the significant transit detection by HATNet, and the positive DS results that exclude obvious false positives, we proceeded with the follow-up of this candidate by obtaining high-resolution and high-S/N spectra to characterize the RV variations and to determine the stellar parameters with higher precision. Using the HIRES instrument (Vogt et al. 1994) on the Keck I telescope located on Mauna Kea, Hawaii, we obtained 22 exposures with an iodine cell, plus one iodine-free template. The observations were made on 16 nights during a number of observing runs between 2007 March 27 and 2008 September 17.

The width of the spectrometer slit used on HIRES was 0 '.86, resulting in a resolving power of $\lambda / \Delta \lambda \approx 55,000$, with a wavelength coverage of $\sim 3800-8000 \AA$. The iodine gas absorption cell was used to superimpose a dense forest of $\mathrm{I}_{2}$ lines on the stellar spectrum and establish an accurate wavelength fiducial (see Marcy \& Butler 1992). Relative RVs in the solar system barycentric frame were derived as described by Butler et al. (1996), incorporating full modeling of the spatial and temporal variations of the instrumental profile. The final RV data and their errors are listed in Table 1. The folded data, with our best fit (see Section 3) superimposed, are plotted in Figure 2. 
Table 1

Relative Radial Velocity, Bisector, and Activity Index Measurements of HAT-P-12

\begin{tabular}{lrcrccc}
\hline \hline $\begin{array}{c}\mathrm{BJD} \\
(2,454,000+)\end{array}$ & $\begin{array}{c}\mathrm{RV}^{\mathrm{a}} \\
\left(\mathrm{m} \mathrm{s}^{-1}\right)\end{array}$ & $\begin{array}{c}\sigma_{\mathrm{RV}}{ }^{\mathrm{b}} \\
\left(\mathrm{m} \mathrm{s}^{-1}\right)\end{array}$ & $\begin{array}{c}\mathrm{BS} \\
\left(\mathrm{m} \mathrm{s}^{-1}\right)\end{array}$ & $\begin{array}{c}\sigma_{\mathrm{BS}} \\
\left(\mathrm{m} \mathrm{s}^{-1}\right)\end{array}$ & $S^{\mathrm{c}}$ & $\sigma_{\mathrm{S}}$ \\
\hline 187.03115 & 46.75 & 3.29 & 2.38 & 4.99 & 0.0063 & 0.00006 \\
187.98977 & -8.83 & 2.74 & -6.80 & 4.45 & 0.0062 & 0.00005 \\
$188.00121^{\mathrm{d}}$ & $\ldots$ & $\ldots$ & 1.64 & 4.97 & 0.0064 & 0.00005 \\
188.10261 & -11.93 & 2.63 & -0.01 & 4.82 & 0.0062 & 0.00005 \\
188.15398 & -20.76 & 3.39 & 2.13 & 5.21 & 0.0059 & 0.00009 \\
188.99152 & -30.42 & 2.43 & -1.37 & 4.82 & 0.0061 & 0.00005 \\
189.07296 & -32.64 & 2.61 & -5.94 & 4.52 & 0.0062 & 0.00006 \\
189.12279 & -19.16 & 2.86 & 3.48 & 5.09 & 0.0066 & 0.00007 \\
216.94159 & -8.61 & 2.68 & -15.51 & 4.19 & 0.0061 & 0.00008 \\
247.86057 & 32.36 & 3.50 & 9.26 & 5.66 & 0.0060 & 0.00011 \\
250.86401 & 20.94 & 3.35 & 9.31 & 5.95 & 0.0057 & 0.00013 \\
251.91230 & 8.95 & 3.09 & 16.91 & 6.31 & 0.0062 & 0.00011 \\
548.03028 & -24.24 & 2.61 & -3.24 & 4.77 & 0.0061 & 0.00006 \\
548.92425 & -24.73 & 3.15 & -4.65 & 4.66 & 0.0059 & 0.00005 \\
602.77839 & -21.39 & 2.29 & -6.79 & 4.64 & 0.0056 & 0.00004 \\
603.02042 & -30.57 & 2.49 & -5.78 & 4.54 & 0.0054 & 0.00006 \\
603.76466 & -7.05 & 2.28 & 0.76 & 5.08 & 0.0057 & 0.00004 \\
604.06935 & -0.63 & 2.84 & 3.57 & 5.40 & 0.0057 & 0.00021 \\
633.94384 & 32.62 & 3.05 & 9.37 & 6.30 & 0.0054 & 0.00018 \\
636.86807 & 39.55 & 2.84 & 13.46 & 6.64 & 0.0060 & 0.00008 \\
639.83599 & 29.74 & 2.92 & -6.83 & 4.50 & 0.0056 & 0.00008 \\
674.76312 & -2.42 & 2.52 & -15.39 & 4.13 & 0.0061 & 0.00006 \\
\hline & & & & & &
\end{tabular}

Notes.

a The fitted zero-point that is on an arbitrary scale has not been subtracted from the velocities.

b The values for $\sigma_{\mathrm{RV}}$ are the formal uncertainties from the spectrum reduction pipeline and do not include our estimate of the stellar jitter.

c This is a relative $S$ index that is sensitive to variations in $S$, it has not been calibrated to the scale of Vaughan et al. (1978).

d This is the iodine-free template exposure for which we do not measure the RV but do measure the BS and $S$ index.

Table 2

Photometry Follow Up of HAT-P-12

\begin{tabular}{lrlll}
\hline \hline $\begin{array}{c}\text { BJD } \\
(2,454,000+)\end{array}$ & \multicolumn{1}{c}{ Mag $^{\mathrm{a}}$} & $\sigma_{\mathrm{Mag}}$ & Mag(orig) $^{\mathrm{b}}$ & Filter \\
\hline 187.74274 & 0.00314 & 0.00073 & 10.51770 & $\mathrm{i}$ \\
187.74377 & 0.00073 & 0.00073 & 10.51630 & $\mathrm{i}$ \\
187.74478 & 0.00086 & 0.00074 & 10.51700 & $\mathrm{i}$ \\
187.74583 & -0.00138 & 0.00072 & 10.51430 & $\mathrm{i}$ \\
187.74683 & 0.00142 & 0.00073 & 10.51610 & $\mathrm{i}$ \\
187.74786 & 0.00369 & 0.00074 & 10.51970 & $\mathrm{i}$ \\
187.74888 & -0.00010 & 0.00073 & 10.51570 & $\mathrm{i}$ \\
187.74991 & 0.00207 & 0.00073 & 10.51720 & $\mathrm{i}$ \\
187.75095 & -0.00404 & 0.00072 & 10.51180 & $\mathrm{i}$ \\
187.75196 & 0.00101 & 0.00072 & 10.51520 & $\mathrm{i}$ \\
\hline
\end{tabular}

Notes.

a The out-of-transit level has been subtracted. These magnitudes have been subjected to EPD and TFA procedure, carried out simultaneously with the transit fit.

$\mathrm{b}$ The raw magnitude values without application of the EPD and TFA procedures.

(This table is available in its entirety in a machine-readable form in the online journal. A portion is shown here for guidance regarding its form and content.)

In Figure 2, we also plot the relative $S$ index. This index is computed following the prescription given by Vaughan et al. (1978) after matching each spectrum to a reference spectrum using a transformation that includes a wavelength shift and a flux scaling that is a polynomial as a function of wavelength. The transformation is determined on regions of the spectra that are

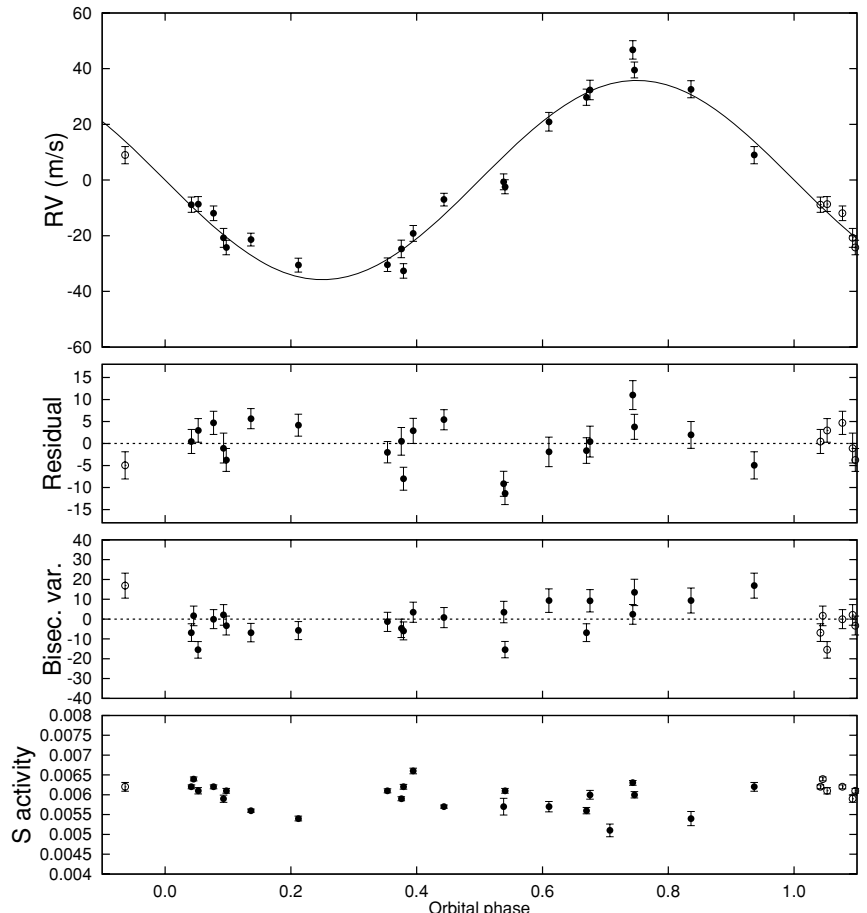

Figure 2. Top: RV measurements from Keck for HAT-P-12, along with an orbital fit, shown as a function of orbital phase, using our best-fit period (see Section 3). Zero phase is defined by the transit midpoint. The center-of-mass velocity has been subtracted. Note that the error bars show the formal errors given by the spectrum reduction pipeline and do not include our estimate of the stellar jitter. Second panel: phased residuals after subtracting the orbital fit (also see Section 3). The rms variation of the residuals is about $4.82 \mathrm{~m} \mathrm{~s}^{-1}$. Third panel: bisector spans (BS) including the template spectrum (Section 3.2). The mean value has been subtracted. Bottom: relative $S$ values for the Keck spectra. Note the different vertical scales of the panels.

not used in computing the $S$ index. Note that the relative $S$ index has not been calibrated to the scale of Vaughan et al. (1978). The relative $S$ index does not show any significant variation correlated with the orbital phase; such a correlation might have indicated that the RV variations are due to stellar activity.

\subsection{Photometric Follow-up Observations}

To confirm the transit signal and obtain high-precision light curves for modeling the system, we conducted photometric follow-up observations with the KeplerCam CCD on the FLWO $1.2 \mathrm{~m}$ telescope. We observed four transit events of HAT-P12b on the nights of 2007 March 27, 2007 April 25, 2009 February 5, and 2009 March 6 (Figure 3). On 2007 March 27,151 frames were acquired with a cadence of $90 \mathrm{~s}(75 \mathrm{~s}$ of exposure time) in the Sloan $i$ band; the observations were interrupted at mid-transit due to clouds. On 2007 April 25, 372 frames were acquired with a cadence of $45 \mathrm{~s}$ (30 s of exposure time) in the Sloan $z$ band. On 2009 February 5, 218 frames were obtained with a cadence of $70 \mathrm{~s}$ (30 s exposure time) in the $z$ band. Finally, on 2009 March 6, 213 frames were acquired with a cadence of $70 \mathrm{~s}$ ( $60 \mathrm{~s}$ exposure time) in the Sloan $g$ band. This follow-up $g$-band light curve was obtained to further constrain possible blend scenarios (Section 3.2).

The reduction of the images was performed as follows. After bias and flat-field calibration, we derived an initial secondorder astrometric transformation between the $\sim 60$ brightest stars and the 2MASS catalog, as described in Pál \& Bakos (2006), yielding a residual of $\sim 0.3-0.4$ pixels. The primary reason for precise astrometry is to minimize the photometric 


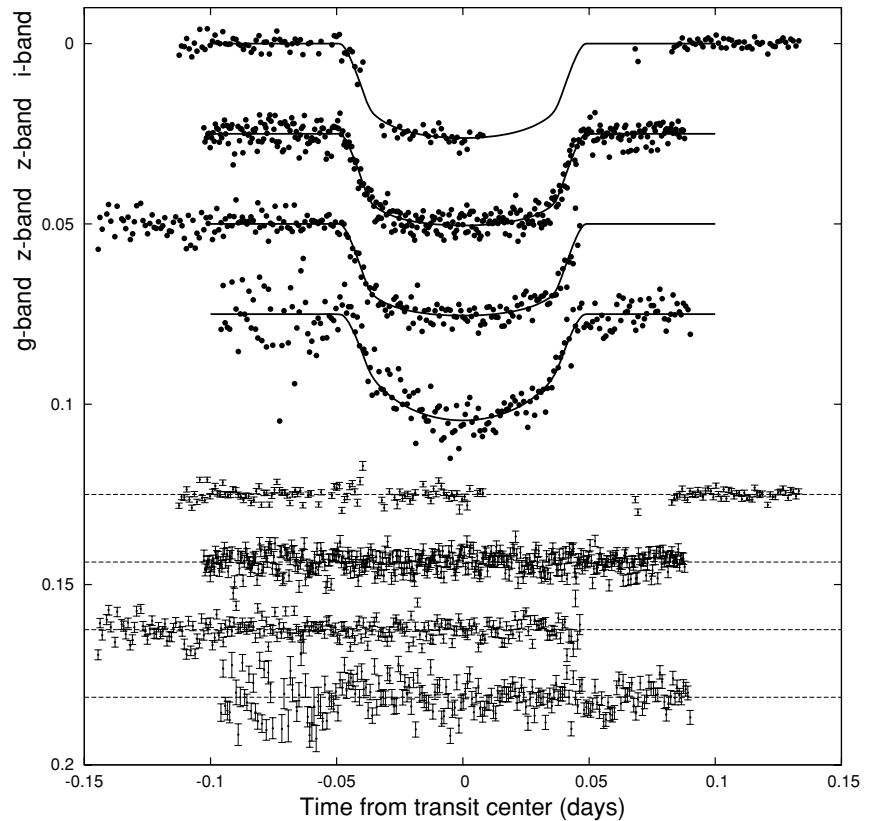

Figure 3. Unbinned instrumental $i-, z-$, and $g$-band transit light curves, acquired with KeplerCam at the FLWO $1.2 \mathrm{~m}$ telescope on the nights of 2007 March 27, 2007 April 25, 2009 February 5, and 2009 March 6 from top to bottom. Superimposed are the best-fit transit model light curves. In the bottom of the figure, we show the residuals from the fit. Error bars represent the photon and background shot noise, plus the readout noise.

errors that would originate from the centroid errors for the individual stars on each frame. Aperture photometry was then performed on the resulting fixed positions, using a series of apertures. The instrumental magnitude transformation was done in two steps: first, all magnitude values were transformed to the photometric reference frame (selected to be the sharpest image), using the individual Poisson noise error estimates as weights. In the second step, the magnitude fit was repeated using the mean individual light curve magnitudes as reference and the rms of these light curves as weights. In both the magnitude transformations, we excluded from the fit the target star itself and the $3 \sigma$ outliers. We performed EPD and TFA against trends simultaneously with the light curve modeling (for more details, see Section 3 and Bakos et al. 2009b). From the series of apertures, for each night, we chose the one yielding the smallest fit rms for the light curve. This aperture conveniently fell in the middle of the aperture series. The final light curves are shown in the upper plots of Figure 3, with our best-fit transit light curve models superimposed (see also Section 3); the photometry is provided in Table 2 .

\section{ANALYSIS}

\subsection{Properties of the Parent Star}

We derived the initial stellar atmospheric parameters by using the template spectrum obtained with the Keck/HIRES instrument. We used the SME package of Valenti \& Piskunov (1996) along with the atomic-line database of Valenti \& Fischer (2005), which yielded the following initial values and uncertainties (which we have conservatively increased to include our estimates of the systematic errors): effective temperature $T_{\text {eff }} \star$ $4650 \pm 60 \mathrm{~K}$, stellar surface gravity $\log g_{\star}=4.75 \pm 0.10$ (cgs), metallicity $[\mathrm{Fe} / \mathrm{H}]=-0.29 \pm 0.05 \mathrm{dex}$, and projected rotational velocity $v \sin i=0.5 \pm 0.4 \mathrm{~km} \mathrm{~s}^{-1}$.

At this stage we could use the effective temperature and the surface gravity as a luminosity indicator, and determine the

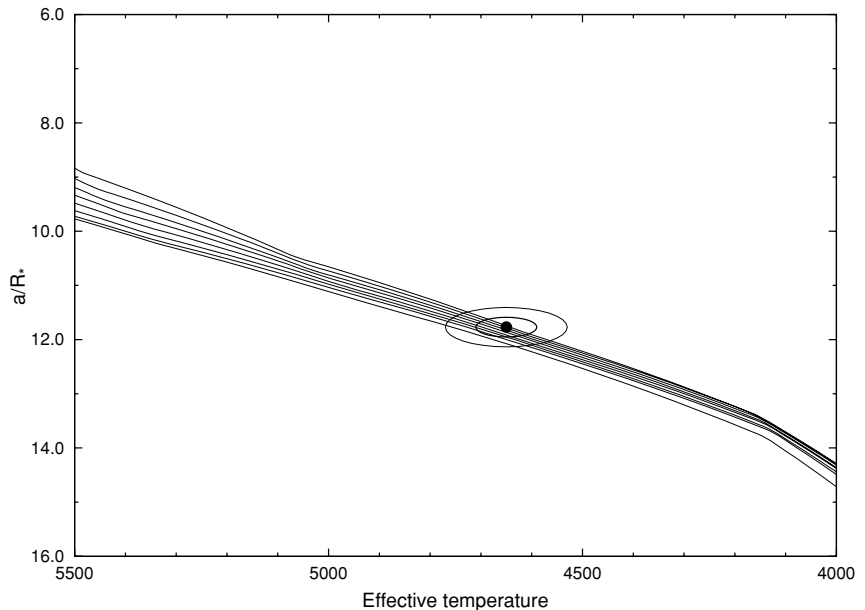

Figure 4. Stellar isochrones from Baraffe et al. $(1997,1998)$ for metallicity $[\mathrm{Fe} / \mathrm{H}]=-0.29$ and ages $0.2,0.5,1.0,2.0,3.0,4.0,5.0$, and $6.0 \mathrm{Gyr}$. The final choice of $T_{\text {eff } \star}$ and $a / R_{\star}$ are marked and encircled by the $1 \sigma$ and $2 \sigma$ confidence ellipsoids.

stellar parameters based on these two constraints using a set of isochrones. However, the effect of $\log g_{\star}$ on the spectral line shapes is typically subtle and as a result it is generally a rather poor luminosity indicator in practice. For planetary transits, the $a / R_{\star}$ normalized semimajor axis and related $\rho_{\star}$ mean stellar density typically impose a stronger constraint on possible stellar models (Sozzetti et al. 2007). The validity of our assumption, namely that the adequate physical model describing our data is a planetary transit (as opposed to a blend), is shown later in Section 3.2.

Using the values of $T_{\mathrm{eff} \star},[\mathrm{Fe} / \mathrm{H}]$, and $\log g_{\star}$ from the SME analysis, and corresponding quadratic limb-darkening coefficients $\left(a_{z}, b_{z}\right.$, etc.) from Claret (2004), we performed a global modeling of the data (Section 3.3), yielding a full Monte Carlo distribution of $a / R_{\star}$. This was complemented by a Monte Carlo distribution of $T_{\text {eff } \star}$ and $[\mathrm{Fe} / \mathrm{H}]$, obtained by assuming Gaussian uncertainties based on the $1 \sigma$ error bars of the initial Spectroscopy Made Easy (SME) analysis.

For each combination within the large $\left(\sim 10^{4}\right)$ set of $a / R_{\star}$, $T_{\text {eff }}$, and $[\mathrm{Fe} / \mathrm{H}]$ values, we searched the stellar isochrones of the Baraffe et al. $(1997,1998)$ models for the best-fit stellar model parameters (such as $M_{\star}, R_{\star}, \log g_{\star}$, etc). We interpolated these isochrones to the SME-based stellar metallicity of $[\mathrm{Fe} / \mathrm{H}]=-0.29 \pm 0.05$. The majority of the parameter combinations in the Monte Carlo search did not match any isochrone. In such cases ( $\sim 60 \%$ of all trials), we skipped to the next randomly drawn parameter set. At the end we derived the mean values and uncertainties of the physical parameters based on their a posteriori distribution. We note that the spread of the input stellar parameters (based on the Gaussian uncertainties) was large compared to what the isochrones cover as a function of age, due to the very slow evolution of K dwarfs (see Figure 4). This is partly the reason for the $40 \%$ match ratio. We also note that the match ratio is very sensitive to changing fundamental parameters of the isochrones, such as the mixing length or the metallicity.

We then repeated the SME analysis by fixing $\log g_{\star}$ to the refined value of $\log g_{\star}=4.61 \pm 0.01$ based on the isochrone search, and only adjusting $T_{\text {eff }},[\mathrm{Fe} / \mathrm{H}]$ and $v \sin i$. This second iteration yielded $T_{\text {eff } \star}=4591 \pm 60 \mathrm{~K},[\mathrm{Fe} / \mathrm{H}]=-0.36 \pm 0.04$, and $v \sin i=1.73 \pm 0.5 \mathrm{~km} \mathrm{~s}^{-1}$. Curiously, the new $T_{\text {eff } \star}$ and $[\mathrm{Fe} / \mathrm{H}]$ values from this second iteration provide a somewhat 
Table 3

Stellar Parameters for HAT-P-12

\begin{tabular}{|c|c|c|}
\hline Parameter & Value & Source \\
\hline$T_{\mathrm{eff} \star}(\mathrm{K})$ & $4650 \pm 60$ & $\mathrm{SME}^{\mathrm{a}}$ \\
\hline$[\mathrm{Fe} / \mathrm{H}]$ & $-0.29 \pm 0.05$ & SME \\
\hline$v \sin i\left(\mathrm{~km} \mathrm{~s}^{-1}\right)$ & $0.5 \pm 0.4$ & SME \\
\hline$v_{\mathrm{mac}}\left(\mathrm{km} \mathrm{s}^{-1}\right)$ & $2.26 \pm 0.0$ & SME \\
\hline$v_{\text {mic }}\left(\mathrm{km} \mathrm{s}^{-1}\right)$ & $0.85 \pm 0.0$ & SME \\
\hline$\gamma_{\mathrm{RV}}\left(\mathrm{km} \mathrm{s}^{-1}\right)$ & $-40.51 \pm 0.21$ & DS \\
\hline$a_{z}$ & 0.3432 & $\mathrm{SME}+$ Claret $^{\mathrm{b}}$ \\
\hline$b_{z}$ & 0.2493 & SME+Claret \\
\hline$a_{i}$ & 0.4323 & SME+Claret \\
\hline$b_{i}$ & 0.2269 & SME+Claret \\
\hline$a_{g}$ & 0.8431 & SME+Claret \\
\hline$b_{g}$ & -0.0064 & SME+Claret \\
\hline$\dot{M}_{\star}\left(M_{\odot}\right)$ & $0.733 \pm 0.018$ & $\mathrm{Baraffe}+a / R_{\star}+\mathrm{SME}^{\mathrm{c}}$ \\
\hline$R_{\star}\left(R_{\odot}\right)$ & $0.701_{-0.012}^{+0.017}$ & Baraffe $+a / R_{\star}+\mathrm{SME}$ \\
\hline $\log g_{\star}(\mathrm{cgs})$ & $4.61 \pm 0.01$ & Baraffe $+a / R_{\star}+\mathrm{SME}$ \\
\hline$L_{\star}\left(L_{\odot}\right)$ & $0.21_{-0.01}^{+0.02}$ & Baraffe $+a / R_{\star}+\mathrm{SME}$ \\
\hline$V(\mathrm{mag})$ & 12.84 & TASS \\
\hline$M_{V}(\mathrm{mag})$ & $6.89 \pm 0.11$ & Baraffe $+a / R_{\star}+\mathrm{SME}$ \\
\hline$K(\mathrm{mag}, \mathrm{CIT})$ & $10.132 \pm 0.017$ & 2MASS+Carpenter ${ }^{\mathrm{d}}$ \\
\hline$M_{K}(\mathrm{mag}, \mathrm{CIT})$ & $4.36 \pm 0.05$ & Baraffe $+a / R_{\star}+\mathrm{SME}$ \\
\hline Age (Gyr) & $2.5 \pm 2.0$ & Baraffe $+a / R_{\star}+\mathrm{SME}$ \\
\hline Distance $(\mathrm{pc})$ & $142.5_{-3.3}^{+4.2}$ & Baraffe $+a / R_{\star}+\mathrm{SME}$ \\
\hline
\end{tabular}

Notes.

" SME = "Spectroscopy Made Easy" package for analysis of high-resolution spectra (Valenti \& Piskunov 1996). These parameters depend primarily on SME, with a small dependence on the iterative analysis incorporating the isochrone search and global modeling of the data, as described in the text.

b $\mathrm{SME}+\mathrm{Claret}=$ Based on the SME analysis and tables of quadratic limbdarkening coefficients from Claret (2004).

${ }^{\mathrm{c}}$ Baraffe $+a / R_{\star}+\mathrm{SME}=$ Baraffe et al. $(1997,1998)$ isochrones, $a / R_{\star}$ relative semimajor axis, and SME results.

${ }^{\mathrm{d}}$ Based on the relations from Carpenter (2001).

inferior match with the Baraffe et al. (1997, 1998) isochrones, as compared to the initial match. Possible reasons for this include (1) systematic errors in the SME analysis due to the relatively low signal-to-noise ratio of our Keck spectra, (2) increasing uncertainty in the SME analysis due to the late stellar type of the host star (note that HAT-P-12 has a temperature that is below the $4700 \mathrm{~K}$ cutoff for stars included in the analysis of Valenti \& Fischer 2005), and (3) general uncertainty in the isochrones for mid-K dwarfs (there is a well-known discrepancy between the observed and predicted mass-radius relation for $\mathrm{K}$ and $\mathrm{M}$ dwarf stars in double-lined eclipsing binaries such that the observed radii are larger than the predicted radii, though there is some evidence that this discrepancy only holds for rapidly rotating, active stars, e.g., Torres \& Ribas 2002; Ribas 2006; López-Morales 2007; Chabrier et al. 2007). Thus, we accepted the initial values of $T_{\mathrm{eff} \star},[\mathrm{Fe} / \mathrm{H}]$, and $v \sin i$ as the final atmospheric parameters for this star, along with the isochrone-based stellar parameters, yielding $M_{\star}=0.733 \pm$ $0.018 M_{\odot}, R_{\star}=0.701_{-0.012}^{+0.017} R_{\odot}$, and $L_{\star}=0.21_{-0.01}^{+0.02} L_{\odot}$. Along with other stellar parameters, these are summarized in Table 3.

The stellar evolutionary isochrones from Baraffe et al. (1997, 1998) for metallicity $[\mathrm{Fe} / \mathrm{H}]=-0.29$ are plotted in Figure 4, with the final choice of effective temperature $T_{\text {eff } \star}$ and $a / R_{\star}$ marked, and encircled by the $1 \sigma$ and $2 \sigma$ confidence ellipsoids.

The stellar evolution modeling also yields the absolute magnitudes and colors in various photometric passbands. We used the apparent magnitudes from the 2MASS catalog (Skrutskie et al. 2006) to determine the distance of the system. The mag- nitudes reported in the 2MASS catalog have to be converted to the CIT system (Elias et al. 1982, 1983), in which the stellar evolution models specify the colors. The reported magnitudes for this star are $J_{2 \mathrm{MASS}}=10.794 \pm 0.023, H_{2 \mathrm{MASS}}=$ $10.236 \pm 0.022$, and $K_{2 \mathrm{MASS}}=10.108 \pm 0.016$; which are equivalent to $J_{\mathrm{CIT}}=10.794 \pm 0.024, H_{\mathrm{CIT}}=10.229 \pm 0.023$, and $K_{\mathrm{CIT}}=10.132 \pm 0.017$; in the CIT photometric system (see Carpenter 2001). Thus, the converted 2MASS magnitudes yield a color of $(J-K)=0.66 \pm 0.03$ that is within $1 \sigma$ of the expected, isochrone-based $(J-K)_{\text {Baraffe }}=0.60 \pm 0.09$. We thus relied on the 2MASS $K$ apparent magnitude and the $M_{\mathrm{K}}=4.36 \pm 0.05$ absolute magnitude derived from the abovementioned modeling to determine the distance: $142.5_{-3.3}^{+4.2} \mathrm{pc}$. The $K$ band was chosen because it is the longest wavelength bandpass with the smallest expected discrepancies due to molecular lines in the spectrum of this K4 dwarf.

\subsection{Excluding Blend Scenarios}

\subsubsection{Spectral Line-Bisector Analysis}

Following Torres et al. (2007), we explored the possibility that the measured RVs are not real, but are instead caused by distortions in the spectral line profiles due to contamination from a nearby unresolved eclipsing binary. A bisector analysis based on the Keck spectra was done as described in Section 5 of Bakos et al. (2007a).

Figure 2 shows the BS phased with the orbital period of the planet. In calculating the BS, we use the convention

$$
\mathrm{BS}=v_{\text {low }}-v_{\text {high }},
$$

where $v_{\text {low }}$ is the velocity of the bisector of the cross-correlation profile at a low cross-correlation value, and $v_{\text {high }}$ is the velocity at a high cross-correlation value. While the BS do not show significant variations with an amplitude that is comparable to or greater than the RV variations, there does appear to be a correlation between the BS and RV measurements. Applying a Spearman rank-order correlation test, we find that the two variables are correlated with $98 \%$ confidence (i.e., there is a $2 \%$ false alarm probability). Since such a correlation might indicate a blend scenario, we consider below, and rule out, the possibility that the system is a blend between a bright foreground $\mathrm{K}$ star and a background eclipsing binary (Section 3.2.2) or a hierarchical triple system (Section 3.2.3). The scenarios that we consider are summarized in Table 4. We then consider the possibility that the correlation is not astrophysical, but rather results from variations in the sky contamination of the spectra (Section 3.2.4); we conclude that this is the most likely explanation for the correlation.

\subsubsection{Contamination from a Background Eclipsing Binary}

The high proper motion of HAT-P-12 allows us to rule out one possible scenario that could potentially fit the available observations, namely a background eclipsing binary that is aligned, by chance, with the foreground K4 dwarf HAT-P-12 (we refer to this as the $\mathrm{H}, \mathrm{b}(\mathrm{S}-\mathrm{s})$ model, where $\mathrm{H}$ stands for the foreground star HAT-P-12, and the $b(\mathrm{~S}-\mathrm{s})$ is a background eclipsing binary star system; here " $b$ " refers to the fact that the eclipsing binary is in the background rather than being associated with the star $\mathrm{H}$ ). To reproduce the observed $\sim 2.5 \%$ deep transit, the background object cannot be more than 4 mag fainter than HAT-P-12 (objects fainter than this would contribute less than $2.5 \%$ of the total combined light and so could not 
Table 4

Blend Configurations

\begin{tabular}{lcc}
\hline \hline Abbreviation & Description & Excluded by \\
\hline H,b(S-s) & Eclipsing binary star diluted by unresolved, unrelated star & Proper motion (Section 3.2.2) \\
H,S-s & Hierarchical triple star system, two components are eclipsing & Light curve fit (Section 3.2.3) \\
H,s-p & Binary star system, fainter star has a transiting planet & Light curve fit (Section 3.2.3) \\
H-p,s & Binary star system, brighter star has a transiting planet & Light curve fit (Section 3.2.3) \\
H-p & Single star with a transiting planet & Not excluded
\end{tabular}
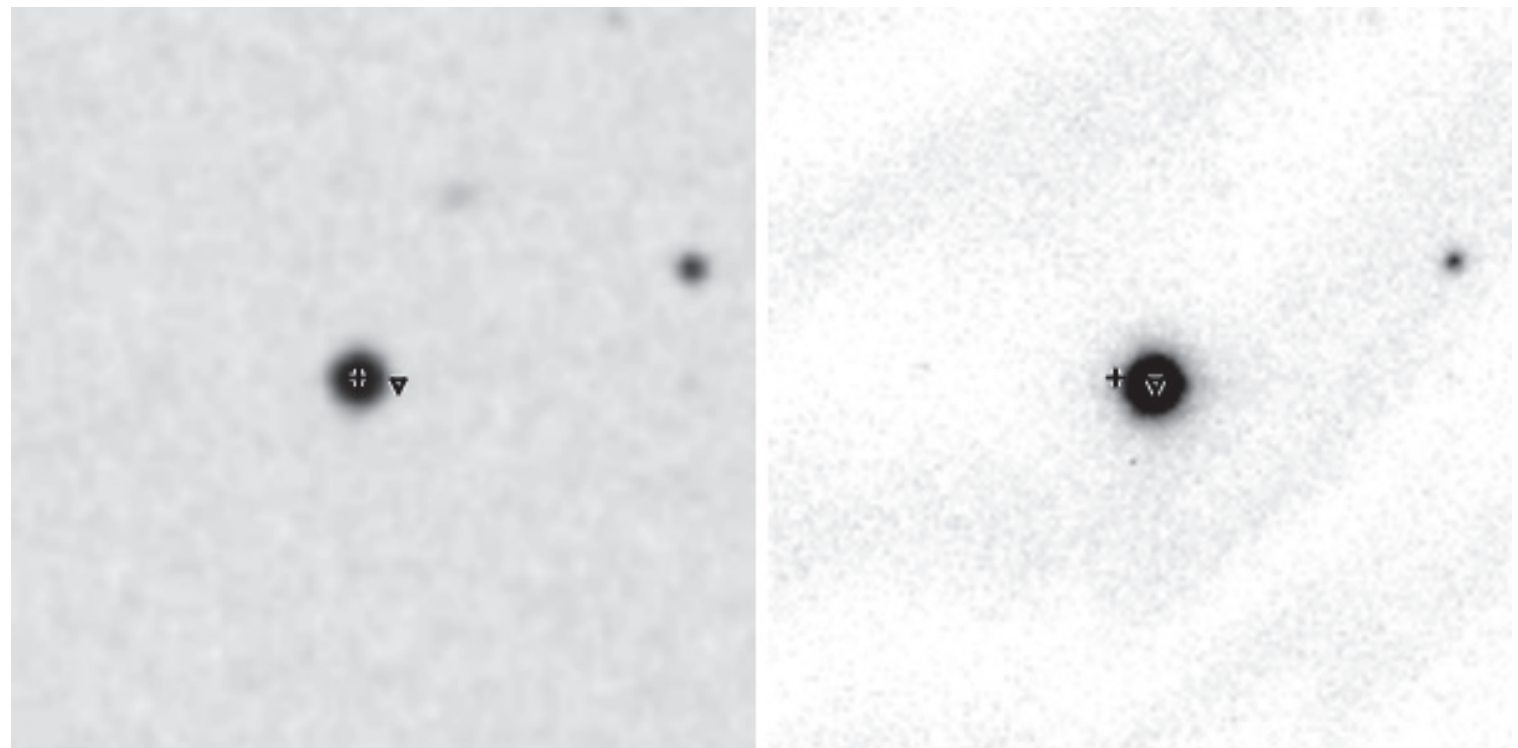

Figure 5. Images of a 2'.5 × 2.5 field containing HAT-P-12 from the POSS-I Red survey (left), and from our FLWO $1.2 \mathrm{~m} z$-band follow-up observations (right, see Section 2.4). The dates of the exposures are 1955 April 13 and 2009 February 5, respectively. The cross marks the position of HAT-P-12 in 1955 and the triangle marks the position in 2009. Between these two dates HAT-P-12 moved 7".5 to the west. From the POSS-I image, we can rule out the presence of stars brighter than $R \sim 19$ at the current position of HAT-P-12.

cause the transit even if they were to be completely eclipsed by an object that emits no light). Because HAT-P-12 has a high

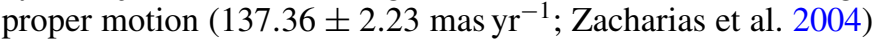
it is possible to use the Palomar Observatory Sky Survey plates from 1955 (POSS-I, red and blue plates) to view the sky at the current position of HAT-P-12 (this same technique was used for HAT-P-11; Bakos et al. 2009b). Between 1955 and the followup observations in 2009, HAT-P-12 has moved 7".5. Figure 5 shows an image stamp from the POSS-I plate compared with a recent observation from the FLWO $1.2 \mathrm{~m}$. We can rule out a background object down to $\sim 19$ mag within $\sim 3^{\prime \prime}$ of the current position of HAT-P-12. Any background object must be $\gtrsim 6 \mathrm{mag}$ fainter than HAT-P-12 and thus could not be responsible for the observed transit.

\subsubsection{Detailed Modeling of a Hierarchical Triple}

Following Bakos et al. (2009b), we consider the possibility that HAT-P-12 is a hierarchical triple system, consisting of two eclipsing bodies that are diluted by a third star. In the following we refer to the bright star, with properties determined from the SME analysis, as HAT-P-12. We consider three scenarios. In the first scenario, we assume that the bright star HAT-P-12 is uneclipsed, and that the two eclipsing components are stars with parameters constrained by common origin to fall on the same age/metallicity isochrone as HAT-P-12 (we refer to this model as the H,S-s model, where $\mathrm{H}$ denotes the bright star HAT-P-12 and S-s denotes a physically associated eclipsing binary consisting of a brighter star $\mathrm{S}$ and a fainter star s). In the second scenario, we assume that HAT-P-12 is uneclipsed, that one of the eclipsing components is a fainter star and that the other component is a planet with negligible mass and luminosity compared to the star (the H,s-p model, where s stands for the fainter star, and $p$ is the planet). In the third scenario, we assume that HAT-P-12 is a star that is transited by a planet and that there is a fainter star diluting the observed transit (the H-p,s model). These models will be compared to the fiducial model of a single star orbited by a planet (the H-p model).

For the H,S-s and H,s-p scenarios, we fit the follow-up z-, $i$-, and $g$-band light curves together with the HATNet $I$-band light curve following the procedure described by Bakos et al. (2009b). We include the HATNet light curve to constrain the possibility of a secondary eclipse; to exclude points that do not contribute to the fit, we only include points that are within one transit duration of the start of transit ingress or end of transit egress, or within one transit duration of the start of secondary ingress or end of secondary egress. We use the TFA HATNet light curve and apply EPD on the out-of-transit portion of the follow-up light curves. We scale the formal photometric errors on each light curve so that $\chi^{2} /$ dof $=1$ for the out-of-transit portion of the light curve. We take the magnitudes and radii of the stars from the Baraffe et al. (1997, 1998) isochrones, transforming the BVRI magnitudes to the Sloan system using the relations from Jordi et al. (2006). To make a fair comparison between the blend models and the H-p model we also fit the H-p model to the light curves using the same procedure used to fit the blend-models (see Section 3.3 for a more detailed analysis of the H-p model used for the final parameter determinations). Figure 6 compares the best-fit H,S-s, H,s-p, and H-p models. 

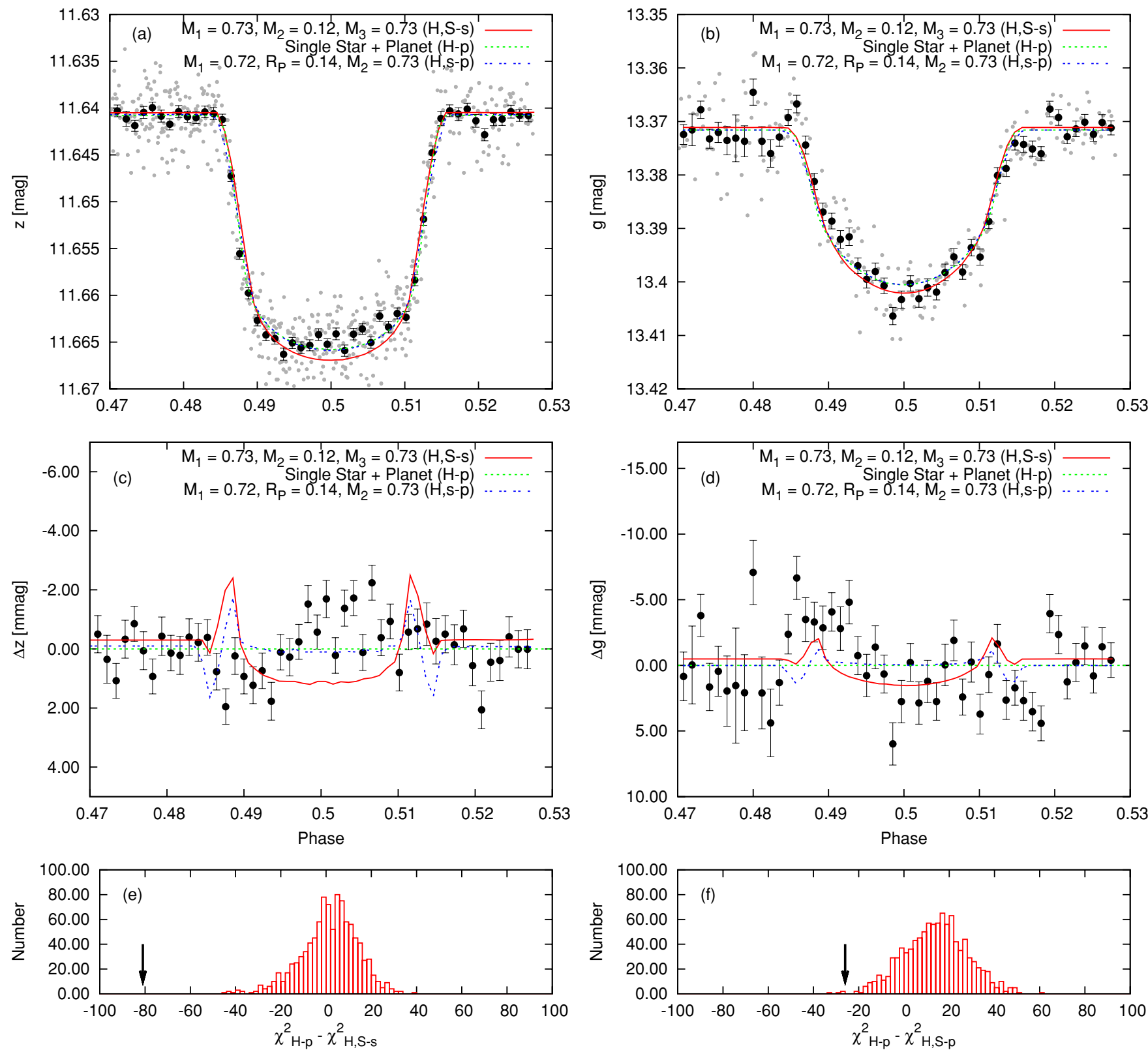

Figure 6. Hierarchical triple blend model fits to the follow-up $z$-band (a) and $g$-band (b) light curves. EPD filtering was performed on the light curves in out-of-transit mode (i.e., a transit model was not simultaneously fit to the light curves). We compare the hierarchical triple models to a model consisting of a single star orbited by a planet. The gray-scale points are the unbinned data, while the dark points are the binned data. Due to time correlations in the noise, the scatter in the binned data is higher than would be expected for white noise (the error bars show the expected errors if the noise were white). To save space we only show the $z$ - and $g$-band light curves, although the follow-up Sloan $i$-band and HATNet $I$-band light curves were also included in the fit. Panels (c) and (d) show the residuals about the best-fit H-p model for the follow-up $z$ - and $g$-band light curves, respectively. Note that the vertical axis for the residual light curves is in mmag. Panels (e) and (f) show the distribution of $\chi_{\mathrm{H}-\mathrm{p}}^{2}-\chi_{\mathrm{H}, \mathrm{S}-\mathrm{S}}^{2}$ and $\chi_{\mathrm{H}-\mathrm{p}}^{2}-\chi_{\mathrm{H}, \mathrm{s}-\mathrm{p}}^{2}$, respectively, from 1000 Monte Carlo simulations of light curves with correlated noise. The arrows mark the observed values. While the best-fit H,S-s and H,s-p models are difficult to distinguish by eye from the best-fit H-p model, they are statistically rejected in favor of the $\mathrm{H}-\mathrm{p}$ model at $\sim 6 \sigma$ and $\sim 3 \sigma$ confidence, respectively.

(A color version of this figure is available in the online journal.)

The best-fit H,S-s model, consisting of an eclipsing pair with masses $M_{1}=0.73 M_{\odot}$ and $M_{2}=0.12 M_{\odot}$ that is diluted by the star HAT-P-12 $\left(M=0.73 \pm 0.02 M_{\odot}\right)$, has $\chi^{2}=1445$ with 1333 degrees of freedom. We compare this to the best-fit H-p model which has $\chi^{2}=1364$ with 1334 degrees of freedom. Because the photometric noise appears to be temporally correlated (Figure 6), formal estimates for the significance of $\Delta \chi^{2}$ between two models will overestimate the confidence with which one model can be rejected in favor of another. We therefore conduct Monte Carlo simulations to estimate the expected distribution of $\Delta \chi^{2}$ values under the assumption that the H-S,s model is correct, and accounting for temporal correlations in the noise. To generate light curves for the Monte Carlo simulations that have similar time-correlated noise as the real light curves, we Fourier transform the residual of each light curve from the best-fit $\mathrm{H}, \mathrm{S}$-s model, randomize the phases, inverse Fourier transform it, and then add in the H,S-s model. This method forces the simulated light curves to have the same noise power spectrum (and hence autocorrelation function) as the actual light curve residuals. We scale the errors of each simulated light curve to have $\chi^{2} /$ dof $=1$ in the out-of-transit portion of the light curve. The Fourier transforms are carried 
out assuming uniform time-sampling (a good approximation for the follow-up light curves); to use the fast Fourier transform algorithm, we cyclically repeat each light curve so that the total number of points in the light curve is a power of 2 . We then fit the H-p and H,S-s models to the simulated sets of light curves and record $\Delta \chi^{2}=\chi_{\mathrm{H}, \mathrm{p}}^{2}-\chi_{\mathrm{H}, \mathrm{S}-\mathrm{s}}^{2}$ for each simulation. From 1000 simulations, we find a median value of $\Delta \chi^{2}=2.41$ with a standard deviation of 12.4 ; we find no instances where $\Delta \chi^{2}<-81$, the minimum value attained is -45 . We conclude that, based on the light curves, the H,S-s model can be rejected in favor of the H-p model at the $\gtrsim 6 \sigma$ confidence level.

For the H,s-p scenario, we find that the best-fit model consists of a star with mass $M_{1}=0.72 M_{\odot}$ transited by a planet with $R_{\mathrm{p}}=1.35 R_{\mathrm{J}}$ and diluted by the star HAT-P-12 $\left(M=0.73 \pm 0.02 M_{\odot}\right)$. This model has $\chi^{2}=1390$ with 1333 degrees of freedom. To determine the significance of $\Delta \chi^{2}=\chi_{\mathrm{H}, \mathrm{p}}^{2}-\chi_{\mathrm{H}, \mathrm{S}-\mathrm{p}}^{2}=-26$ we repeat the Monte Carlo simulations, this time adopting the best-fit H,s-p model as the fiducial model. From 1000 simulations, we find a median value of $\Delta \chi^{2}=14.3$ with a standard deviation of 13.9. There are four simulations with $\Delta \chi^{2} \leqslant-26$, so we conclude that the $\mathrm{H}$ $\mathrm{p}$ model is preferred over the H,s-p model with $99.6 \%(\lesssim 3 \sigma)$ confidence.

As described in Section 3.3, it is possible to correct for systematic errors in the photometry by simultaneously applying EPD and TFA to the light curves while fitting a physical model to them. By using a more sophisticated model of this form we are able to rule out the H,s-p model with higher confidence, and also rule out the H-p,s model. We perform the global modeling as described in Section 3.3 incorporating three additional parameters that allow for dilution in the $g, i$, and $z$ bands. This model effectively encompasses both the H-p,s and H,s-p models because the only H,s-p models that provide a reasonable fit to the light curve are models where the planetbearing star has a mass that is nearly equal to the mass of the diluting star HAT-P-12. We allow the dilution factors to vary independently in the fit. We find that models with no dilution are strongly preferred, and place $1 \sigma$ upper limits on the light contribution in each filter from an uneclipsed star ("third light") of $l_{3, z}<3 \%, l_{3, i}<3.5 \%$, and $l_{3, g}<5 \%$. Any additional star thus makes a negligible contribution to the total light of the system. This test thus rules out both the H-p,s and H,s-p models.

\subsubsection{Bisector Variations Induced by Sky Contamination}

As shown in Sections 3.2.3 and 3.2.2, blend scenarios involving an eclipsing binary star system are inconsistent with other observations of the system. We therefore look for an explanation of the apparent BS-RV correlation shown in Section 3.2.1 that does not invoke a blend. One possibility is that it is due to varying contamination from the sky spectrum. Because HAT-P-12 is relatively faint, the flux from the sky is non-negligible compared to the flux from the source. Scattered light from the moon illuminating the sky near HAT-P-12 has a solar-like spectrum which yields a peak near $\mathrm{RV}=0 \mathrm{~km} \mathrm{~s}^{-1}$ in the cross-correlation profile of the observed spectrum. The degree to which this second peak contaminates the peak from the star varies with the sky brightness and the RV difference between the moon and the star. Because the observer-centric velocity of HAT-P-12 is always less than the velocity of the moon, an increase in the sky brightness or a decrease in the velocity difference will lead to a positive $B S$ variation for our adopted BS sign convention.
While the sky brightness is not directly measurable from the available data, in order to quantify this effect we may introduce a sky contamination factor (SCF) given by

$$
\mathrm{SCF}=\frac{I}{\Delta V^{2}+\left(\frac{1}{2} \Gamma\right)^{2}},
$$

where $I$ is the ratio of the flux in the spectrum due to the moon to the flux due to the star, $\Delta V$ is the observer-centric RV difference between the moon and the star, $\Gamma=15.28 \mathrm{~km} \mathrm{~s}^{-1}$ is the width of the Lorentzian function that best fits the mean cross-correlation profile, and the form for the denominator is chosen because the cross-correlation profile is well fit by a Lorentzian function. We estimate $I$ via the relation

$$
I=\frac{T_{0} / t_{0}}{T / t} 10^{-0.4\left(B_{m}-B_{S}\right)},
$$

where $T$ is the total flux received in the region of the spectrum used to compute the BS, $t$ is the exposure time, $T_{0}$ and $t_{0}$ are the values for the spectrum with the highest count rate (these are used to account for changes in the flux received from the star due to variations in the seeing or transparency), $B_{S}$ is the $B$-band magnitude of the star (we take $B_{S}=13.84$ assuming $(B-V) \sim 1.0$ for a dwarf star with $\left.T_{\text {eff }}=4650 \mathrm{~K}\right)$, and $B_{m}$ is the effective magnitude of the sky due to the moon at the position of the star (in an area of $\sim 2.5 \operatorname{arcsec}^{2}$ ). To estimate $B_{m}$ we use the model for the sky brightness due to moonlight given by Krisciunas \& Schaefer (1991), extending it to the $B$ band by taking $(B-V)=0.91$ for the moon (e.g., Schaefer 1998) and $k=0.19$ for the extinction coefficient (this is a typical value at the summit of Mauna Kea for the Johnson $B$-band which is roughly the region of the spectrum used to calculate the BS; Krisciunas et al. 1987). The values for $B_{m}$ range from 18.15 to 19.86 . When the moon is below the horizon we take $B_{m}=99.99$.

Figure 7 compares the SCF to the BS values and to the orbital phase. Note that we normalize the SCF to have a mean value of 1.0. There is a positive correlation between the SCF and the BS. By chance, spectra taken between orbital phases 0.5 and 1 had higher sky contamination on average than those taken between orbital phases 0 and 0.5 . When points with $\mathrm{SCF}>1.0$ are removed, the correlation between the remaining $\mathrm{BS}$ and $\mathrm{RV}$ values is no longer significant (the correlation significance is $37 \%$ ). We conclude that this a plausible explanation for the apparent BS-RV correlation.

As a further test on this hypothesis, we simulate sky contaminated spectra and measure the BS values using the same procedure as for the actual spectra. To simulate a spectrum we take

$$
s_{i}=z\left(t, V_{t}-V_{i}\right)+I \times z\left(t_{2}, V_{t_{2}}-\gamma_{t_{2}}\right),
$$

where $t$ is the iodine-free template spectrum of HAT-P-12, $t_{2}$ is the iodine-free template spectrum of HAT-P-13 scaled to have the same total flux though the $B$-band as $t$ (HAT-P-13 has $T_{\text {eff }}=5638 \mathrm{~K}$, and is thus a better approximation to a solar spectrum than HAT-P-12; Bakos et al. 2009c), $V_{t}$ and $V_{t_{2}}$ are the barycentric velocity corrections for the templates, $V_{i}$ is the barycentric velocity correction for spectrum $i, \gamma_{t_{2}}$ is the average RV of HAT-P-13, $I$ is given by Equation (3), and $z(x, y)$ is a function that redshifts the spectrum $x$ by velocity $y$. Figure 8 compares the SCF to the BS for the simulated spectra. The simulations show a correlation between the SCF and BS that is comparable to that seen in Figure 7(a). This confirms that sky contamination may affect the BS values at the level that is observed. 

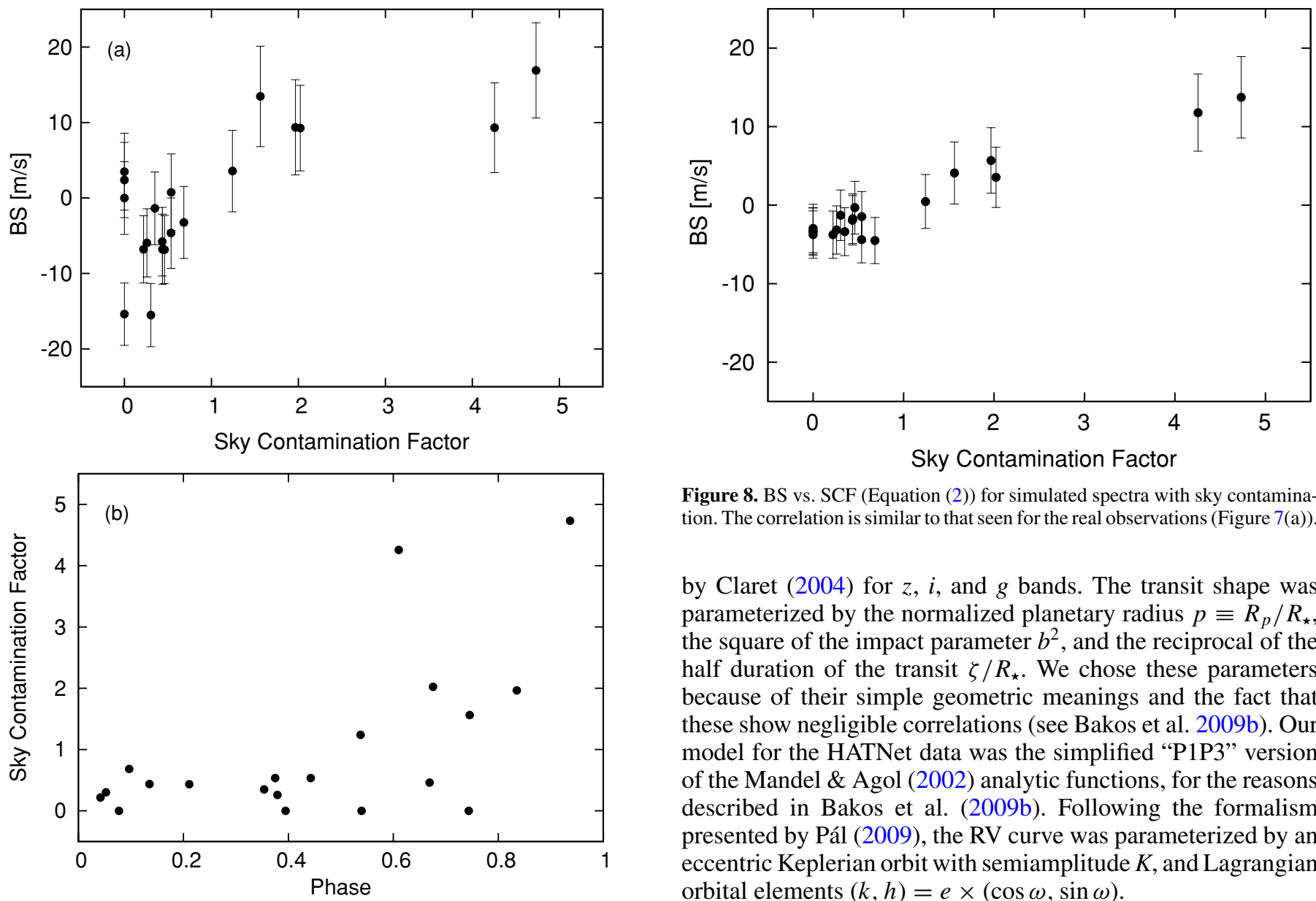

Figure 8. BS vs. SCF (Equation (2)) for simulated spectra with sky contamination. The correlation is similar to that seen for the real observations (Figure 7(a)).

by Claret (2004) for $z, i$, and $g$ bands. The transit shape was parameterized by the normalized planetary radius $p \equiv R_{p} / R_{\star}$, the square of the impact parameter $b^{2}$, and the reciprocal of the half duration of the transit $\zeta / R_{\star}$. We chose these parameters because of their simple geometric meanings and the fact that these show negligible correlations (see Bakos et al. 2009b). Our model for the HATNet data was the simplified "P1P3" version of the Mandel \& Agol (2002) analytic functions, for the reasons described in Bakos et al. (2009b). Following the formalism presented by Pál (2009), the RV curve was parameterized by an eccentric Keplerian orbit with semiamplitude $K$, and Lagrangian orbital elements $(k, h)=e \times(\cos \omega, \sin \omega)$.

We assumed that there is a strict periodicity in the individual

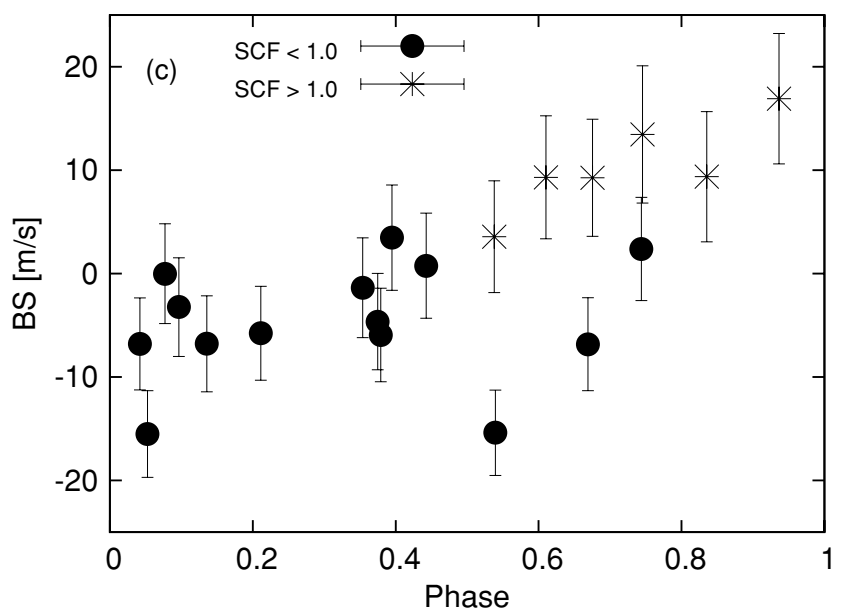

Figure 7. (a) BS vs. SCF (Equation (2)). The two variables appear to be positively correlated. (b) SCF vs. orbital phase. Spectra taken between phases 0.5 and 1 had by chance higher sky contamination on average than the spectra taken between phases 0 and 0.5 . As a result, the BS are correlated with the orbital phase. (c) BS vs. orbital phase shown separately for points with high and low SCF. When points with high SCF are removed, there is no longer an apparent correlation between BS and orbital phase.

\subsection{Global Modeling of the HAT-P-12 System}

Our model for the follow-up light curves used analytic formulae based on Mandel \& Agol (2002) for the eclipse of a star by a planet, where the stellar flux is described by quadratic limb darkening. The limb-darkening coefficients were derived from the SME results (Section 3.1), using the tables provided transit times. In practice, we assigned the transit number $N_{\mathrm{tr}}=0$ to the first high quality follow-up light curve gathered on 2007 March 27. The adjusted parameters in the fit were the first transit center observed by HATNet, $T_{c,-132}$, and the last transit center observed by the FLWO $1.2 \mathrm{~m}$ telescope, $T_{c,+212}$, covering all of our measurements with the HATNet telescopes, and the FLWO $1.2 \mathrm{~m}$ telescope. We prefer using $T_{c,-132}$ and $T_{c,+212}$ as adjusted parameters rather than the period and epoch for the reasons discussed by Bakos et al. (2007c) and Pál et al. (2008). The transit center times for the intermediate transits were interpolated using these two epochs and the $N_{\text {tr }}$ transit number of the actual event. The model for the RV data contains the ephemeris information through the $T_{c,-132}$ and $T_{c,+212}$ variables (Pál 2009). Altogether, the 11 parameters describing the physical model were $T_{c,-132}, T_{c,+212}, R_{p} / R_{\star}, b^{2}, \zeta / R_{\star}, K$, $k=e \cos \omega, h=e \sin \omega$, and three additional ones related to the instrumental configuration. These are the instrumental blend factor $B_{\text {inst }}$ of HATNet which accounts for possible dilution of the transit in the HATNet light curve, the HATNet out-of-transit magnitude, $M_{0, \mathrm{HATNet}}$, and the relative RV zero-point $\gamma_{\text {rel }}$.

We extended our physical model with an instrumental model that describes the systematic variations of the data. This was done in a similar fashion to the analysis presented in Bakos et al. (2009b). Basically, the HATNet photometry has been already EPD- and TFA-corrected before the global modeling, so we only considered systematic corrections to the followup light curves. We chose the "ELTG" method, i.e., EPD was performed in "local" mode with EPD coefficients defined for each night, and TFA was performed in "global" mode using the same set of stars and TFA coefficients for all nights. 
The underlying physical model was based on the Mandel \& Agol (2002) analytic formulae, as described earlier. The five EPD parameters were the hour angle (characterizing a monotonic trend that changes linearly over time), the square of the hour angle, and the stellar profile parameters (equivalent to FWHM, elongation, position angle). The exact functional form of the above parameters contained six coefficients, including the auxiliary out-of-transit magnitude of the individual events. The EPD parameters were independent for all four nights, implying 24 additional coefficients in the global fit. For the global TFA analysis we chose 18 template stars that had good quality measurements for all nights and on all frames, implying an additional 18 parameters in the fit. We apply EPD to the template star light curves using the same set of parameters as used for the HAT-P-12 light curves before incorporating them in the analysis. Thus, the total number of fitted parameters is 11 $($ physical model $)+24$ (local EPD) +18 (global TFA $)=53$, i.e., much smaller than the number of data points $(\gtrsim 1000)$.

The joint fit was performed as described in Bakos et al. (2009b). We minimized $\chi^{2}$ in the parameter space by using a hybrid algorithm, combining the downhill simplex method (AMOEBA; see Press et al. 1992) with the classical linear least-squares algorithm. Uncertainties on the parameters were derived using the Markov Chain Monte Carlo method (MCMC; see Ford 2006) using "Hyperplane-CLLS" chains (Bakos et al. 2009b). The a priori distributions of the parameters for these chains were chosen from a generic Gaussian distribution, with eigenvalues and eigenvectors derived from the Fisher covariance matrix for the best-fit value. The Fisher covariance matrix is calculated analytically using the partial derivatives given by Pál $(2008,2009)$. Since the eccentricity of the system appeared as insignificant $(k=0.052 \pm 0.025, h=0.007 \pm 0.046)$, we repeated the global fit by fixing these to 0 . The best-fit results for the relevant physical parameters are summarized in Table 5. Table 5 also lists the RV "jitter," which is a component of assumed astrophysical noise intrinsic to the star that we add in quadrature to the RV measurement uncertainties in order to have $\chi^{2} /$ dof $=1$ from the RV data for the global fit. In addition, some auxiliary parameters (not listed in the table) were $T_{\mathrm{c},-132}=$ $2453792.64889 \pm 0.00044(\mathrm{BJD}), T_{\mathrm{c},+212}=2454897.94147 \pm$ 0.00038 (BJD), and $\gamma_{\text {rel }}=32.6 \pm 1.3 \mathrm{~m} \mathrm{~s}^{-1}$ (for the Keck RVs, note that this does not correspond to the true center of mass $\mathrm{RV}$ of the system, but is only a relative offset). The planetary parameters and their uncertainties can be derived by the direct combination of the a posteriori distributions of the light curve, $\mathrm{RV}$ and stellar parameters. We found that the mass of the planet is $M_{p}=0.211 \pm 0.012 M_{\mathrm{J}}=67.02 \pm 3.71 M_{\oplus}$, the radius is $R_{p}=0.959_{-0.021}^{+0.029} R_{\mathrm{J}}=10.75_{-0.24}^{+0.32} R_{\oplus}$ and its density is $\rho_{p}=0.295 \pm 0.025 \mathrm{~g} \mathrm{~cm}^{-3}$. The final planetary parameters are summarized at the bottom of Table 5 .

\section{DISCUSSION}

Comparing HAT-P-12b to the theoretical models of Baraffe et al. (2008), we find that the mass and radius of the planet are consistent with the $1.0 \mathrm{Gyr}, Z=Z_{\odot}=0.02$ nonirradiated model, or with a 1.0-5.0 Gyr, $0.10<Z<0.50$ irradiated model (Figure 9). With an equilibrium temperature of $T_{\text {eq }}=963 \pm 16 \mathrm{~K}$, HAT-P-12b has an equivalent solar semimajor axis of $a_{\text {equiv }}=0.084 \mathrm{AU}$, so the irradiation received by the planet, while not insignificant, is less than what is used to calculate the irradiated model $\left(a_{\text {equiv }}=0.045 \mathrm{AU}\right)$. The inferred metal fraction is expected to be closer to $Z=0.10$
Table 5

Orbital and Planetary Parameters

\begin{tabular}{lc}
\hline \multicolumn{1}{c}{ Parameter } & Value \\
\hline Light curve parameters & \\
$P($ days $)$ & $3.2130598 \pm 0.0000021$ \\
$T_{c}(\mathrm{BJD})$ & $2454419.19556 \pm 0.00020$ \\
$T_{14}(\text { days })^{\mathrm{a}}$ & $0.0974 \pm 0.0006$ \\
$T_{12}=T_{34}(\text { days })^{\mathrm{a}}$ & $0.0125 \pm 0.0005$ \\
$a / R_{\star}$ & $11.77_{-0.21}^{+0.15}$ \\
$\zeta / R_{\star}$ & $23.57 \pm 0.12$ \\
$R_{p} / R_{\star}$ & $0.1406 \pm 0.0013$ \\
$b^{2}$ & $0.044_{-0.024}^{+0.035}$ \\
$b \equiv a \cos i / R_{\star}$ & $0.211_{-0.078}^{+0.066}$ \\
$i(\mathrm{deg})$ & $89.0 \pm 0.4$ \\
$\mathrm{RV}$ parameters & \\
$K\left(\mathrm{~m} \mathrm{~s}^{-1}\right)$ & $35.8 \pm 1.9$ \\
$k_{\mathrm{RV}} \mathrm{b}^{\mathrm{b}}$ & 0 \\
$h_{\mathrm{RV}}{ }^{\mathrm{b}}$ & 0 \\
$e$ & 0 \\
$\mathrm{RV}$ jitter $\left(\mathrm{m} \mathrm{s}^{-1}\right)$ & 4.82 \\
$\mathrm{Planetary} \mathrm{parameters}$ & \\
$M_{p}\left(M_{\mathrm{J}}\right)$ & $0.211 \pm 0.012$ \\
$R_{p}\left(R_{\mathrm{J}}\right)$ & $0.959_{-0.021}^{+0.029}$ \\
$C\left(M_{p}, R_{p}\right)^{\mathrm{c}}$ & 0.63 \\
$\rho_{p}\left(\mathrm{~g} \mathrm{~cm}{ }^{-3}\right)$ & $0.295 \pm 0.025$ \\
$a(\mathrm{AU})$ & $0.0384 \pm 0.0003$ \\
$\log g_{p}\left(\mathrm{cgss}^{-3}\right.$ & $2.75 \pm 0.03$ \\
$T_{\mathrm{eq}}(\mathrm{K})$ & $963 \pm 16$ \\
$\Theta^{\mathrm{d}}$ & $0.023 \pm 0.001$ \\
$\langle F\rangle\left(10^{12} \mathrm{erg} \mathrm{s}^{-1} \mathrm{~cm}^{-2}\right)^{\mathrm{e}}$ & $1.91 \pm 0.12$ \\
\hline &
\end{tabular}

Notes.

${ }^{a} T_{14}$ : total transit duration, time between first and last contact; $T_{12}=T_{34}$ : ingress/egress time, time between first and second, or third and fourth contact.

${ }^{\mathrm{b}}$ Fixed to 0 .

${ }^{c}$ Correlation coefficient between the planetary mass $M_{p}$ and radius $R_{p}$.

$\mathrm{d}$ The Safronov number is given by $\Theta=\frac{1}{2}\left(V_{\text {esc }} / V_{\text {orb }}\right)^{2}=$ $\left(a / R_{p}\right)\left(M_{p} / M_{\star}\right)$ (see Hansen \& Barman 2007).

${ }^{\mathrm{e}}$ Incoming flux per unit surface area.

than $Z=0.50$ for the Baraffe et al. (2008) models if the correct irradiation were used.

We have also compared HAT-P-12b to the theoretical models of Fortney et al. (2007). In Figure 9 we have interpolated these models to $a_{\text {equiv }}=0.084 \mathrm{AU}$, and find that the mass and radius of HAT-P-12b are consistent with a $10 M_{\oplus}$ core, 1 Gyr model, and lie between the coreless and $10 M_{\oplus}$ core, $4.5 \mathrm{Gyr}$ models. We conclude, therefore, that HAT-P-12b is most likely an $\mathrm{H} / \mathrm{He}$-dominated planet with a core of perhaps $\lesssim 10 M_{\oplus}$, and a total metal fraction of $\lesssim 15 \%$. This makes HAT-P-12b the least massive $\mathrm{H} / \mathrm{He}$-dominated gas giant planet found to date; the previous record holder was Saturn.

It is interesting to compare the properties of HAT-P-12b to those of Saturn and HD 149026b, the two planets with known radii that have masses closest to that of HAT-P-12b. Measurements of the mass of HD $149026 \mathrm{~b}$ range from $0.36 M_{\mathrm{J}}$ to $0.37 M_{\mathrm{J}}$, while determinations of its radius range from $0.65 R_{\mathrm{J}}$ to $0.813 R_{\mathrm{J}}$ (Sato et al. 2005; Charbonneau et al. 2006; Torres et al. 2008; Winn et al. 2008; Nutzman et al. 2009; Carter et al. 2009). The planet appears to have a significant core, with estimates ranging from $45 M_{\oplus}$ to $114 M_{\oplus}$ (see Carter et al. 2009, and references therein), implying a high metal fraction of $Z \gtrsim 0.4$. Saturn has a mass of $0.299 M_{\mathrm{J}}$ (Standish 1995), equatorial radius 




Figure 9. Mass-radius diagram of TEPs (filled squares; blue for those found by HATNet and red for those found by other surveys) and solar system planets (triangles). HAT-P-12b is shown as a large filled square on the left. Overlaid are the Baraffe et al. (2008) irradiated planetary isochrones for ages of 0.5, 1.0, and 5.0 Gyr and metal fractions of $Z=0.1$ and $Z=0.5$, the Baraffe et al. (2008) non-irradiated, 1.0 Gyr, solar metallicity isochrone, and the Fortney et al. (2007) 1.0 Gyr and 4.5 Gyr isochrones for planets with core masses of $M_{C}=0$ and $M_{C}=10 M_{\oplus}$ interpolated to the solar-equivalent semimajor axis of HAT-P-12b. We also show the isodensity lines for $0.4,0.7,1.0,1.33,5.5$, and $11.9 \mathrm{~g} \mathrm{~cm}^{-3}$ (dashed lines). HAT-P-12b appears to be well modeled as a 1.0 $4.5 \mathrm{Gyr}$, mildly irradiated planet with a core mass of $M_{C} \lesssim 10 M_{\oplus}$. HAT-P-12b is the lowest-mass $\mathrm{H} / \mathrm{He}$-dominated gas giant planet found to date.

(A color version of this figure is available in the online journal.)

of $0.843 R_{\mathrm{J}}$ (Seidelmann et al. 2007), an estimated core mass of $9 M_{\oplus} \lesssim M_{C} \lesssim 22 M_{\oplus}$, and a total heavy element fraction of $0.14 \lesssim Z \lesssim 0.29$ (Saumon \& Guillot 2004). Although HAT$\mathrm{P}-12 \mathrm{~b}$ is less massive than both HD $149026 \mathrm{~b}$ and Saturn, it has a larger radius than both planets. Note that HAT-P-12b does not have a detectable eccentricity, so its large radius may not be due to tidal heating (in the models by Jackson et al. 2008, however, close-in planets may have tidally inflated radii even with eccentricities $\lesssim 0.01$ ). The large radius in comparison with Saturn may be due in part to the enhanced irradiation received by HAT-P-12b, and to HAT-P-12b potentially having a smaller core mass than Saturn. HD 149026b, on the other hand, receives more irradiation than HAT-P-12b $\left(a_{\text {equiv }}=0.025 \mathrm{AU}\right.$ using the parameters from Carter et al. 2009), so the difference in radii suggests that HAT-P-12b has a substantially smaller core mass and metal enhancement than HD 149026b.

It is interesting to note that the inferred core mass of the three planets appears to correlate with the host star metallicity (HAT$\mathrm{P}-12$ has $[\mathrm{Fe} / \mathrm{H}]=-0.29 \pm 0.05$, the Sun has $[\mathrm{Fe} / \mathrm{H}]=0$, and HD 149026 has $[\mathrm{Fe} / \mathrm{H}]=0.36 \pm 0.05$ from Sato et al. 2005). This correlation has been previously noted by Guillot et al. (2006) and Burrows et al. (2007), and is perhaps suggestive evidence for the core accretion model of planet formation (e.g., Alibert et al. 2005, and references therein). Further discoveries of TEPs with masses comparable to or less than that of Saturn are needed to determine whether or not this correlation holds. Note from Figure 9 that the radii of planets in this mass regime are more sensitive to the core mass than are the radii of more massive planets for which a given core mass is a smaller fraction of the total planet mass.

Finally, one might wonder why other planets like HAT-P-12b have not been found to date (see Figure 9). The significant $\sim 2.5 \%$ transit depth of HAT-P-12b is well within the range that is easily detectable for many transit surveys, and the $35.8 \pm 1.9 \mathrm{~km} \mathrm{~s}^{-1} \mathrm{RV}$ semiamplitude, while small, is still easily measured with high-precision RV spectrometers (though more observations may be needed for a robust confirmation, which may slow the rate at which these planets are announced). We conclude that of hot gaseous planets with radii similar to Jupiter, only a small fraction have masses similar to Saturn such as HAT-P-12b; the majority have masses similar to Jupiter. With the discovery of HAT-P-12b, we estimate that the fraction is $\sim 2 \%$, with considerable uncertainty.

We thank the referee, Peter McCullough, for several suggestions that improved the quality of this paper, and Scott Gaudi for a helpful discussion. HATNet operations have been funded by NASA grants NNG04GN74G, NNX08AF23G, and SAO IR\&D grants. Work of G.Á.B. and J. Johnson were supported by the Postdoctoral Fellowship of the NSF Astronomy and Astrophysics Program (AST-0702843 and AST-0702821, respectively). We acknowledge partial support also from the Kepler Mission under NASA Cooperative Agreement NCC21390 (D.W.L., PI). G.K. thanks the Hungarian Scientific Research Foundation (OTKA) for support through grant K-60750. This research has made use of Keck telescope time granted through NOAO and NASA. The Digitized Sky Surveys were produced at the Space Telescope Science Institute under U.S. Government grant NAG W-2166. The images of these surveys are based on photographic data obtained using the Oschin Schmidt Telescope on Palomar Mountain and the UK Schmidt Telescope. The plates were processed into the present compressed digital form with the permission of these institutions. This research has made use of the SIMBAD database, operated at CDS, Strasbourg, France.

\section{REFERENCES}

Alibert, Y., Mordasini, C., Benz, W., \& Winisdoerffer, C. 2005, A\&A, 434, 343 Bakos, G. Á., Noyes, R. W., Kovács, G., Stanek, K. Z., Sasselov, D. D., \& Domsa, I. 2004, PASP, 116, 266

Bakos, G. Á., et al. 2007a, ApJ, 670, 826

Bakos, G. Á., et al. 2007b, ApJ, 670, 826

Bakos, G. Á., et al. 2007c, ApJ, 671, L173

Bakos, G. Á., et al. 2009a, ApJ, 696, 1950

Bakos, G. Á., et al. 2009b, arXiv:0901.0282

Bakos, G. Á., et al. 2009c, ApJ, in press (arXiv:0907.3525)

Baraffe, I., Chabrier, G., Allard, F., \& Hauschildt, P. H. 1997, A\&A, 327, 1054

Baraffe, I., Chabrier, G., Allard, F., \& Hauschildt, P. H. 1998, A\&A, 337, 403

Baraffe, I., Chabrier, G., \& Barman, T. 2008, A\&A, 482, 315

Burke, C. J., et al. 2007, ApJ, 671, 2115

Burrows, A., Hubeny, I., Budaj, J., \& Hubbard, W. B. 2007, ApJ, 661, 502

Butler, R. P., et al. 1996, PASP, 108, 500

Carpenter, J. M. 2001, AJ, 121, 2851

Carter, J. A., Winn, J. N., Gilliland, R., \& Holman, M. J. 2009, ApJ, 696, 241

Chabrier, G., Gallardo, J., \& Baraffe, I. 2007, A\&A, 472, L17

Charbonneau, D., Brown, T. M., Noyes, R. W., \& Gilliland, R. L. 2002, ApJ, 568,377

Charbonneau, D., et al. 2006, ApJ, 636, 445

Claret, A. 2004, A\&A, 428, 1001

Deleuil, M., Deeg, H. J., Alonso, R., Bouchy, F., \& Rouan, D. 2008, A\&A, 491, 889

Droege, T. F., Richmond, M. W., \& Sallman, M. 2006, PASP, 118, 1666

Elias, J. H., Frogel, J. A., Hyland, A. R., \& Jones, T. J. 1983, AJ, 88, 1027

Elias, J. H., Frogel, J. A., Matthews, K., \& Neugebauer, G. 1982, AJ, 87, 1029

Fabrycky, D. C., \& Winn, J. N. 2009, ApJ, 696, 1230

Ford, E. 2006, ApJ, 642, 505

Fortney, J. J., Marley, M. S., \& Barnes, J. W. 2007, ApJ, 659, 1661

Gillon, M., et al. 2007, A\&A, 472, L13

Gillon, M., et al. 2009, A\&A, 501, 785

Guillot, T., Santos, N. C., Pont, F., Iro, N., Melo, C., \& Ribas, I. 2006, A\&A, 453, L21

Hansen, B. M. S., \& Barman, T. 2007, ApJ, 671, 861

Jackson, B., Greenberg, R., \& Barnes, R. 2008, ApJ, 681, 1631

Johns-Krull, C. M., et al. 2008, ApJ, 677, 657 
Jordi, K., Grebel, E. K., \& Ammon, K. 2006, A\&A, 460, 339

Knutson, H. A., Charbonneau, D., Allen, L. E., Burrows, A., \& Megeath, S. T. 2008, ApJ, 673, 526

Kovács, G., Bakos, G. Á., \& Noyes, R. W. 2005, MNRAS, 356, 557

Kovács, G., Zucker, S., \& Mazeh, T. 2002, A\&A, 391, 369

Krisciunas, K., \& Schaefer, B. E. 1991, PASP, 103, 1033

Krisciunas, K., et al. 1987, PASP, 99, 887

Latham, D. W. 1992, in ASP Conf. Ser. 32, IAU Coll. 135, Complementary Approaches to Double and Multiple Star Research, ed. H. A. McAlister \& W. I. Hartkopf (San Francisco, CA: ASP), 110

Léger, A., et al. 2009, A\&A, in press (arXiv:0908.0241)

López-Morales, M. 2007, ApJ, 660, 732

Mandel, K., \& Agol, E. 2002, ApJ, 580, L171

Mandushev, G., et al. 2007, ApJ, 667, L195

Marcy, G. W., \& Butler, R. P. 1992, PASP, 104, 270

Nutzman, P., Charbonneau, D., Winn, J. N., Knutson, H. A., Fortney, J. J., Holman, M. J., \& Agol, E. 2009, ApJ, 692, 229

Pál, A. 2008, MNRAS, 390, 281

Pál, A. 2009, MNRAS, 396, 1737

Pál, A., \& Bakos, G. Á. 2006, PASP, 118, 1474

Pál, A., et al. 2008, ApJ, 680, 1450

Pont, F., Bouchy, F., Queloz, D., Santos, N. C., Melo, C., Mayor, M., \& Udry, S. 2004, A\&A, 426, L15

Press, W. H., Teukolsky, S. A., Vetterling, W. T., \& Flannery, B. P. 1992 Numerical Recipes in C: The Art of Scientific Computing (2nd ed.; Cambridge: Cambridge Univ. Press)
Ribas, I. 2006, Ap\&SS, 304, 89

Sato, B., et al. 2005, ApJ, 633, 465

Saumon, D., \& Guillot, T. 2004, ApJ, 609, 1170

Schaefer, B. E. 1998, skytel, 95, 57

Seager, S., Kuchner, M., Hier-Majumder, C. A., \& Militzer, B. 2007, ApJ, 669 , 1279

Seidelmann, P. K., et al. 2007, Celest. Mech. Dyn. Astron., 98, 155

Skrutskie, M. F., et al. 2006, AJ, 131, 1163

Sozzetti, A., et al. 2007, ApJ, 664, 1190

Standish, E. M. 1995, Highlights Astron., 10, 180

Torres, G., Boden, A. F., Latham, D. W., Pan, M., \& Stefanik, R. P. 2002, AJ, 124,1716

Torres, G., \& Ribas, I. 2002, ApJ, 567, 1140

Torres, G., Winn, J. N., \& Holman, M. J. 2008, ApJ, 677, 1324

Torres, G., et al. 2007, ApJ, 666, 121

Valenti, J. A., \& Fischer, D. A. 2005, ApJS, 159, 141

Valenti, J. A., \& Piskunov, N. 1996, A\&AS, 118, 595

Vaughan, A. H., Preston, G. W., \& Wilson, O. C. 1978, PASP, 90, 267

Vogt, S. S., et al. 1994, Proc. SPIE, 2198, 362

West, R. G., et al. 2009a, A\&A, 502, 395

West, R. G., et al. 2009b, AJ, 137, 4834

Winn, J. N., Henry, G. W., Torres, G., \& Holman, M. J. 2008, ApJ, 675, 1531

Winn, J. N., et al. 2005, ApJ, 631, 1215

Zacharias, N., Monet, D. G., Levine, S. E., Urban, S. E., Gaume, R., \& Wycoff, G. L. 2004, BAAS, 36, 1418 